\title{
Phytoprotection
}

\section{Liste des arbitres 1993-1995}

\section{List of Reviewers 1993-1995}

Volume 77, numéro 2, 1996

URI : https://id.erudit.org/iderudit/706105ar

DOI : https://doi.org/10.7202/706105ar

Aller au sommaire du numéro

\section{Éditeur(s)}

Société de protection des plantes du Québec (SPPQ)l

\section{ISSN}

0031-9511 (imprimé)

1710-1603 (numérique)

Découvrir la revue

Citer ce document

(1996). Liste des arbitres 1993-1995. Phytoprotection, 77(2), 93-95.

https://doi.org/10.7202/706105ar d'utilisation que vous pouvez consulter en ligne.

https://apropos.erudit.org/fr/usagers/politique-dutilisation/ 


\section{Liste des arbitres 1993-1995 List of Reviewers 1993-1995}

Le Comité de rédaction de PHYTOPROTECTION veut reconnaître l'excellente contribution de nombreux collaborateurs qui ont généreusement participé au processus d'évaluation des manuscrits. Qu'ils trouvent ici l'expression de nos remerciements les plus sincères.

The Editorial Board of PHYTOPROTECTION would like to acknowledge the excellent contribution of many collaborators who generously participated in the evaluation of manuscripts. May they find here the expression of our most sincere gratitude.

Abbas, H.K.

Alex, J.

Amfer, B.

Anderson, T.R.

Andrews, J.H.

Asselin, A.

Beauchamp, C.J.

Beaulieu, C.

Bergstrom, G.

Bernier, L.

Blackshaw, R.

Boland, G.J.

Bouchard, C.J.

Boyetchko, S.

Brodeur, J.

Buchwaldt, L.

Cardina, J.

Cavers, P.B.

Cloutier, C.

Cloutier, D.

Cossentine, J.E.

Couture, L.

Crozier, L.

Derksen, D.

Devaux, A.

Dion, $\mathrm{P}$.
United States Dept. of Agriculture, Stoneville, Mississippi

University of Guelph, Guelph, Ontario

Georgia Experimental Station, Griffin, Georgia

Agriculture and Agri-Food Canada, Harrow, Ontario

University of Wisconsin, Madison, Wisconsin

Université Laval, Québec

Université Laval, Québec

Université de Sherbrooke, Sherbrooke, Québec

Cornell University, Ithaca, New York

Université Laval, Québec

Agriculture and Agri-Food Canada, Lethbridge, Alberta

University of Guelph, Guelph, Ontario

Ministère de I'Agriculture, des Pêcheries et de l'Alimentation du Québec, Sainte-Foy, Québec

Agriculture and Agri-Food Canada, Regina, Alberta

Université Laval, Québec

University of Manitoba, Winnipeg, Manitoba

Ohio State University, Columbus, Ohio

University of Western Ontario, London, Ontario

Université Laval, Québec

Agriculture et Agroalimentaire Canada, L'Assomption, Québec

Agriculture and Agri-Food Canada, Summerland, British Columbia

Agriculture et Agroalimentaire Canada, Sainte-Foy, Québec

Nova Scotia Dept. of Agriculture, Truro, Nova Scotia

Agriculture and Agri-Food Canada, Brandon, Manitoba

Ministère de l'Agriculture, des Pêcheries et de l'Alimentation du Québec, Saint-Hyacinthe, Québec

Université Laval, Québec 
Dostaler, D.

Ellis, C.

Fields, $\mathrm{P}$.

Gealy, D.

Gilbert, G.

Gleason, M.L.

Gossen, B.

Grafius, E.J.

Hedges, B.R.

Hildebrand, P.D.

Hogue, R.

Holliday, N.J.

Holt, J.

Hsiang, $T$.

Hunt, D.W.

Innes, L.

Isman, M.

Jamieson, A.R.

Jarvis, W.

Jensen, K.I.N.

Jones, $\mathrm{R}$.

Kimpinski, J.

Kirkland, K.J.

Köller, W.

Le Blanc, J.-P.R.

Lemieux, $\mathrm{C}$.

Leroux, G.D.
Université Laval, Québec

University of Guelph, Guelph, Ontario

Agriculture and Agri-Food Canada, Winnipeg, Manitoba

United States Dept. of Agriculture-ARS, Stuttgart, Arizona

Ministère de l'Agriculture, des Pêcheries et de l'Alimentation du Québec, Sainte-Foy, Québec

lowa State University, Ames, lowa

Agriculture and Agri-Food Canada, Saskatoon, Saskatchewan

Michigan State University, East Lansing, Michigan

Agriculture and Agri-Food Canada, Harrow, Ontario

Agriculture and Agri-Food Canada, Kentville, Nova Scotia

Ministère de l'Agriculture, des Pêcheries et de l'Alimentation du Québec, Sainte-Foy, Québec

University of Manitoba, Winnipeg, Manitoba

University of California, Riverside, California

University of Guelph, Guelph, Ontario

Agriculture and Agri-Food Canada, Harrow, Ontario

Ministère des Ressources naturelles, Québec, Québec

University of British Columbia, Vancouver, British Columbia

Agriculture and Agri-Food Canada, Kentville, Nova Scotia

Agriculture and Agri-Food Canada, Harrow, Ontario

Agriculture and Agri-Food Canada, Kentville, Nova Scotia

North Carolina State University, Raleigh, North Carolina

Agriculture and Agri-Food Canada, Charlottetown, Prince Edward Island

Agriculture and Agri-Food Canada, Scott, Saskatchewan

Cornell University, Ithaca, New York

Nova Scotia Agricultural College, Truro, Nova Scotia

Agriculture et Agroalimentaire Canada, Sainte-Foy, Québec

Université Laval, Québec

Agriculture and Agri-Food Canada, Regina, Saskatchewan

lowa State University, Ames, lowa

Horticultural Research Institute, Vineland, Ontario

Agriculture and Agri-Food Canada, Winnipeg, Manitoba

University of Illinois, Illinois

Agriculture et Agroalimentaire Canada, Sainte-Foy, Québec

Agriculture and Agri-Food Canada, Ottawa, Ontario

University of Nebraska, Nebraska

New Brunswick Dept. of Agriculture, Fredericton, New Brunswick 
Olthof, T.H.A.

Otrysko, B.

Ouellette, G.

Peterson, R.L.

Potter, J.W.

Rahe, J.E.

Richard, C.
Agriculture and Agri-Food Canada, Vineland, Ontario Ministère de l'Agriculture, des Pêcheries et de l'Alimentation du Québec, Les Buissons, Québec

Centre de foresterie des Laurentides, Sainte-Foy, Québec

University of Guelph, Guelph, Ontario

Agriculture and Agri-Food Canada, Vineland, Ontario

Simon Fraser University, Vancouver, British Columbia

Agriculture et Agroalimentaire Canada, Sainte-Foy, Québec

Rodriguez-Kabana, R. Auburn University, Auburn, Alabama

Saindou, G.

Agriculture and Agri-Food Canada, Lethbridge, Alberta

Schoen, D.

McGill University, Montréal, Québec

Scriven, R.W.D.

University of California, Davis, California

Seabrook, B. University of New Brunswick, Moncton, New Brunswick

Smalley, E.B.

Smith, R.

University of Wisconsin, Madison, Wisconsin

Agriculture and Agri-Food Canada, Kentville, Nova Scotia

Souza-Machado, V.

Stephenson, G.

Stewart, J.

Sutton, $\mathrm{J}$.

Sweeney, J.

Tekauz, A.

Thomas, A.G.

Tomlin, A.D.

Thurstar, G.

Traquair, J.

Turgeon, J.

Vanden Born, W.

Warwick, S.

Weaver, S.E.

Webster, R.

Wiles, L.J.

Wise, I.

Woodrow, L.

Zhang, J.
University of Guelph, Guelph, Ontario

University of Guelph, Guelph, Ontario

Agriculture and Agri-Food Canada, Charlottetown, Prince Edward Island

University of Guelph, Guelph, Ontario

Forestry Canada, Fredericton, New Brunswick

Agriculture and Agri-Food Canada, Winnipeg, Manitoba

Agriculture and Agri-Food Canada, Regina, Saskatchewan

Agriculture and Agri-Food Canada, London, Ontario

University of California, Davis, California

Agriculture and Agri-Food Canada, London, Ontario

Forestry Canada, Sault Ste. Marie, Ontario

University of Alberta, Edmonton, Alberta

Agriculture and Agri-Food Canada, Ottawa, Ontario

Agriculture and Agri-Food Canada, Harrow, Ontario

Fredericton, New Brunswick

United States Dept. of Agriculture-ARS, Fort Collins, Colorado

Agriculture and Agri-Food Canada, Winnipeg, Manitoba

Agriculture and Agri-Food Canada, Harrow, Ontario

Agriculture and Agri-Food Canada, Harrow, Ontario 